\title{
高 $T_{c}$ 氧化物超导体中的氧缺位和 局部位形激发模式*
}

\author{
熊 诗 杰 \\ （中国高等科学技术中心理论物理分中心，南京大学物理系、固体物理䂨究所）
}

在某些复合化物中所发现的高温超导电性 ${ }^{[1-3]}$, 引起了新的探索超越 BCS 理论的超导 机制的热潮.对 $\mathrm{La}_{2-x} \mathrm{Ba}_{x} \mathrm{CuO}$, 系统所进行的电子能带结构的计算 $[4,5]$ 表明电声子机制有可能 仍对这种系统中所发现的 30 40K 的超导电性起着主导的作用. 对其 Elishberg 函数和电声 子㧴合常数的计算 ${ }^{[6]}$ 进一步支持了这种观点. 但这种理论仍然难以解释新近在 Y-Ba-Cu-O. 系统中所发现的液氮温区的超导电性. 现在已证实 ${ }^{[3]}$, 大量的形如 $\mathrm{ABa}_{2} \mathrm{Cu}_{3} \mathrm{O}_{6+x}$ 的化合物系 统 (其中 $\mathrm{A}$ 为 $\mathrm{Y}, \mathrm{La}, \mathrm{Nd}, \mathrm{Sm}$ 等元素), 都具有高于 $90 \mathrm{~K}$ 的超导电性. 在五元化合物 $\mathrm{Y}-$ $\mathrm{Ba}-\mathrm{Cu}-\mathrm{F}-\mathrm{O}$ 系统中甚至发现了 $155 \mathrm{~K}$ 的超导电性 ${ }^{[2)}$. 所有这些再加上新近对 $\mathrm{YBa}_{2} \mathrm{Cu}_{3} \mathrm{O}$ ，和 $\mathrm{EuBa}_{2} \mathrm{Cu}_{3} \mathrm{O}$, 系统所进行的同位素效应的研究结果 ${ }^{\left[{ }^{2}\right]}$, 都对传统的 $\mathrm{BCS}$ 电声子机制和其他非 传统的电声子机制(如双极化子机制 ${ }^{[10)}$ )提出了严峻的挑战.

本文基于对这一类氧化物中由氧缺位所引起的局部位形的分析，提出了一种由局部位形 激发模式产生的电子配对机制. 通过对计算结果的分析对进一步提高 $T_{\mathrm{c}}$ 的途径做了一些预测.

对于 $\mathrm{x}<3$ 的 $\mathrm{ABa}_{2} \mathrm{Cu}_{3} \mathrm{O}_{6+x}$ 形的化合物系统, 与完整的钻钓矿结构相比, 或多或少地存 在着一些氧的缺位. 如果以一个铜氧八面体为基本的局部位形单位, 那末根据氧缺位的数目, 氧八面体可以退化成方锥, 四面体或平面四方形. 相应的铜离子也取不同的羍化态一 $\mathrm{Cu}^{+}$, $\mathrm{Cu}^{++}, \mathrm{Cu}^{+++}$. 为简单计, 我们只考虑从面体中移走一个原子的局部位形. 对于 $\mathrm{ABa}_{2}$. $\mathrm{Cu}_{3} \mathrm{O}_{6+x}$ 系统, 在整个晶格骨架中氧八面体是在基面上收缩的, 因此对一个氧缺位, 具有两种 不同的基本结构位形,一种是氧缺位在基面上,另一种是氧缺位不在基面上. 二者具有不同的 能量. 二者之间的转换对应着氧缺位在不同位置之间的跳跃, 需要克服一定的势垒. 我们把 这种转换看作是系统的一种基本的元敨发模式, 称作局部位形激发, 记作 LSE. 与电声子互作 用类似, 电子与 LSE 也有互作用. 由于 LSE 实际上是对应着氧原子在不同格位上的跳跃, 它应伴随着周围离子的振动, 也就是多声子过程.

考虑到 LSE 系统后,系统的哈密顿可写成

$$
H=H_{c}+H_{p h}+H_{c-p h}+H_{s}+H_{c-s}+H_{c-e},
$$

这里 $H_{c}$ 和 $H_{p h}$ 分别是自由电子和声子的哈密顿, $H_{e-p h}$ 和 $H_{e-c}$ 分别是电声子互作用和电 子间的库仑互作用, $H_{s}$ 是 LSE 系统的哈密顿, $H_{c-s}$ 则是电子和 LSE 的互作用. 如果用 Pauli 矩阵表征不同局部位形之间的转换,则 $H_{s}$ 可写成

$$
H_{s}=-Q \sum_{i} \sigma_{i}^{*}-\frac{1}{2} \sum_{\langle i, i\rangle} J \sigma_{j}^{z} \sigma_{i}^{x},
$$


这里求和对基本位形单位进行. $Q$ 是两种不同局部位形之间的能量差, $J$ 是近邻位形单位之 间 LSE 的有效耦合. 哈密顿中的互作用部分是

$$
\begin{aligned}
& H_{e-p h}=\sum_{p, p^{\prime}, \lambda} V_{p h}\left(\boldsymbol{p}, \boldsymbol{p}^{\prime}, \lambda\right) a_{p^{\prime}}^{+} a_{p^{\prime}}\left(b_{p-p^{\prime}, \lambda}+b_{p^{\prime}-p, \alpha}^{+1}\right), \\
& H_{c-s}=\sum_{\boldsymbol{p}, \boldsymbol{p}^{\prime}} V_{s}\left(\boldsymbol{p}, \boldsymbol{p}^{\prime}\right) a_{\boldsymbol{p}^{+}}^{+} a_{\boldsymbol{p}^{\prime}} i \sigma_{\boldsymbol{p}-\boldsymbol{p}^{\prime}}^{y}, \\
& H_{e-c}=\sum_{p, p^{\prime}, q} V_{c}(\boldsymbol{q}) a_{p^{+}+\boldsymbol{q}^{+}}^{+} a_{p^{\prime}-q^{+} a_{p^{\prime}} a_{p},},
\end{aligned}
$$

这里 $a_{p}$ 和 $b_{q, \lambda}$ 分别是波矢为 $\boldsymbol{p}$ 的电子和波矢为 $\boldsymbol{q}$ 、极化方向为 $\lambda$ 的声子的消灭算符. $V_{c}$ （q）是电子间的屏蔽库仑势, 而 $V_{p h}$ 及 $V$, 可写成

$$
\begin{gathered}
V_{p h}\left(\boldsymbol{p}, \boldsymbol{p}^{\prime}, \lambda\right)=-i\left(N_{0} / 2 m \omega_{02}\left(\boldsymbol{p}-\boldsymbol{p}^{\prime}\right)\right)^{1 / 2} \boldsymbol{e}_{\boldsymbol{p}-\boldsymbol{p}^{\prime}, \lambda} \cdot\left\langle\boldsymbol{p}^{\prime}|\nabla V(\boldsymbol{x})| \boldsymbol{p}\right\rangle, \\
V_{s}\left(\boldsymbol{p}, \boldsymbol{p}^{\prime}\right)=N^{1 / 2}\left\langle\boldsymbol{p}^{\prime}|\nabla V(\boldsymbol{x})| \boldsymbol{p}\right\rangle \cdot \boldsymbol{s} \Lambda,
\end{gathered}
$$

这里 $V_{p h}$ 是标准的电声子耦合系数的形式, 对于 $V_{s}$, 我们记人了伴随着局部位形激发的多 声子过程 $\Lambda, 8$ 则是在局部位形激发中氧原子的位移. $N_{0}$ 和 $N$ 分别是氧原子和氧蚗位的数 目. 如果位形激发所要克服的势垒是 $W$,则 $\Lambda$ 可近似地看成是一个统计因子:

$$
\Lambda \sim \exp \left(-W / k_{B} T\right),
$$

它反映了跨越势垒的变换几率.

与电声子耦合类似，电子-LSE 耦合也可以产生电子的配对效应. 为了比较二者在超导 中所起的作用,我们研究两个耦合系数之比:

$$
t=\left|V_{s}\left(p, p^{\prime}\right)\right|^{2} /\left|V_{p h}\left(p, p^{\prime}\right)\right|^{2} \sim 2 \exp \left(-2 W / k_{B} T\right) s^{2} m \omega_{0} N / N_{0} .
$$

对形如 $\mathrm{ABa}_{2} \mathrm{Cu}_{3} \mathrm{O}_{6+x}$ 的化合物, 如 $x \sim 1$, 则有 $N / N_{0} \sim 2 / 7, s$ 的数值约在 $2.5 \AA \sim 3.5 \AA$ 之 间, $\infty_{0}$ 是声子的特征频率. 我们把它看作是氧八面体的呼吸模, 近似地取作 $40 \mathrm{meV}$, 于是有

$$
t \sim 350 \exp \left(-2 W / k_{B} T\right) \text {. }
$$

这样, 只要统计因子 $\exp \left(-2 W / k_{B} T\right)$ 不是太小, 则 LSE 表现出与传导电子的极强的耦合。 这主要的于在局部位形激发过程中氧原子的大的位移. 从统计因子的形式可以看出, 温度越 高, LSE 起的作用就越大, 因此我们有理由期待这种通过 LSE 的电子配对机制在高温区超 导电性中的主导作用.

从上面的讨论可以看出, 与电声子耦合不同，电子-LSE 耦合是与氧原子的质量无关的. 两个重要的参数-能量差 $\Omega$ 和势垒 $W$ 主要取决于结构、空间电位分布及离子的电荷. 因此, 如 果 LSE 的作用是主导的, 将不会观察到明显的同位素效应, 这与文献 [9]实验结果一致.

为了进一步分析 LSE 在提高超导转变温度中所起的作用，我们采用一般的强耦合理论， 用类似于文献[11]中的方法得到了在同时计及电声子耦合和电子-LSE 耦合时计算超导转变 温度 $T_{c}$ 的公式. 在超强耦合极限, $T_{c}$ 由如下方程给出

$$
\begin{aligned}
2 \int_{0}^{\infty} \omega d \omega & \left(\left(\alpha_{p h}^{2}(\omega) F_{p h}(\omega)+\alpha_{s}^{2}(\omega) F_{s}(\omega)\right) /\left(\omega^{2}+\left(2 \pi T_{c} k_{B}\right)^{2}\right)\right) \\
& -2 \mu^{*}+1,
\end{aligned}
$$

这里 $\alpha_{p h}^{2}(\omega) F_{p h}(\omega)$ 和 $\alpha_{s}^{2}(\omega) F_{s}(\omega)$ 分别是声子和 LSE 的 Eliashberg 函数. 对于 LSE 系 统, 在不同温度下得到的谱密度由图 1 所示, 可以看出在温度升高时谱密度的峰变窄, 但峰的 位置不变. 在图 2 中, 我们给出了对不同的 $W$ 值所算出的超导转变温度 $T_{c}$ 随 $Q$ 值变化的曲 线. 从图中可以看出, 在 $\Omega$ 值较低时, LSE 系统对超导没有影响, $T_{c}$ 取只有电声子耦合时的 
数值,在 $\Omega$ 值超过某一临界值时, $T_{c}$ 才突然上升，表现出 LSE 对超导的主导作用. 这一临 界值随着 $W$ 的加大而增大,而对同样的 $\Omega$ 值, $W$ 的增大却使 $T_{c}$ 下降.

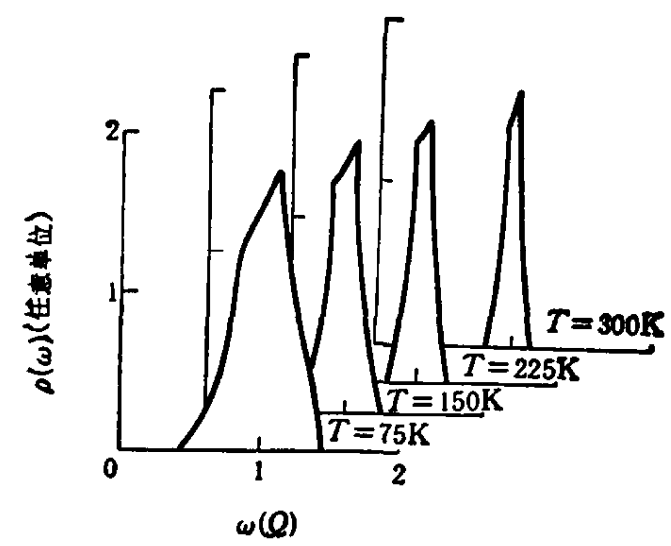

图 1 在不同温度下 LSE 系统的㙕密度曲线 其他参数估是: $Q=6.5 \mathrm{meV}$, $J=0.65 \mathrm{meV}$

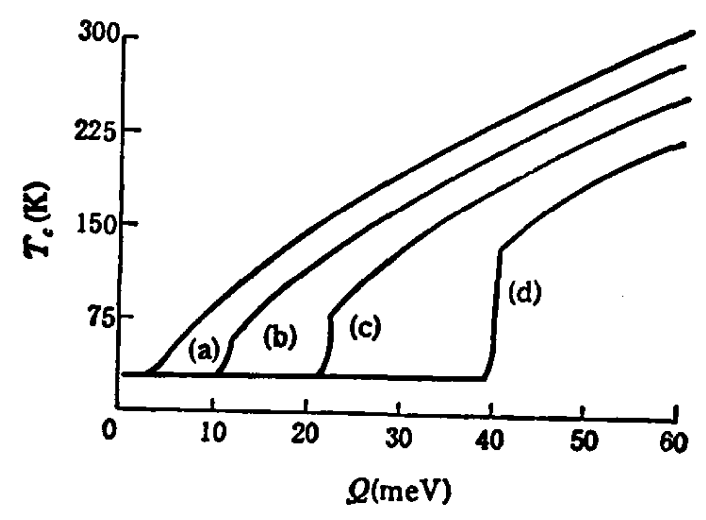

图 2 对不同的 $w$ 值,超导转变温度随 0 值变化的曲线 $t \sim 350 \exp \left(-2 \mathrm{~W} / k_{\mathrm{B}} J\right), \mu^{*}=0.1$. (a) $W=50 \mathrm{meV}$; (b) $\mathrm{W}=100 \mathrm{meV}$; (c) $W=125 \mathrm{meV}$; (d) $W=200 \mathrm{meV}$

以上结果是十分有趣的. 根据这样的计算结果, 可以设想如下的进一步提高 $T$ c 的途径:

（1）减小势垒 $W$. 一个可以想见的办法是减小在局部位形激发中移动的原子的半径, 因 为半径越小的原子在原子间穿行越容易. 比氧离子半径更小的负离子有氧离子等, 实验已证 实五元化合物 Y-Ba-Cu-F-O 确实具有更高的 $T_{c}^{\text {[8] }}$, 说明这种途径是可行的. $W$ 的数值还 与空间结构、电位分布及键的强度等因素有关. 详细的讨论将另文进行.

（2）增大 $\Omega$ 值. $Q$ 是两种局部结构的能量差, 因此氧八面体在基面方向和轴向上的差距 越大,就越有利. 用四元化合物来代替三元化合物,或使整个晶格结构准二维化, 都提供了增 大样八面体各向异性的机会. 这些做法在实验上都是行之有效的.

（3）控制氧的化学配比,使样品中有一定比例的氧缺位存在.

本文的一些基本结论与已有的实验结果都是吻合的, 没有发现严重的矛盾. 但仍需进一 步直接的、严格的实验结果证实. 希望本文的一些结果能引起更多实验和理论工作的兴趣.

致谢：作者感谢冯端教授、拱昌德教授、本正中教授、徐龙道教授等在工作中的支持和有益的讨论.

[ 1] Bednorz, J. G. and Muller, K. A., Z. Phys, B84(1986), 189.

[2]制忠贤、陈立息等, 科学通报, 32(1987), 6: 412 .

[ 3 ] Chu, C. W, Hor, P. H. et al., Phys. Rev. Lett, 58(1987), 405.

[4] Mattheiss, L. F. Phys. Rev. Lett, 58 (1987), 1028.

[5] Xu, J. H., Watson-Yang, T. J. et al., Phys. Lett, A120 (1987), 489.

(6) Weber, W., Phys. Rev. Lezt, 58(1987), 1371.

[ 7 ] Hor, P. H., Meng, R. L. et al., Phys. Rev. Letz, 58(1987), 1891.

[ 8 ] Ovshinsky, S. R., Young, R. T. et al., Phys. Rev. Lett, 58(1987), 2579.

[9] Batlogg, B. et al., Phys. Rev. Letz. (in press).

[10] Mott, N. F., Nature, 327(1987), 185.

[11] Allea, P. B, Dynes, R. C., Phys. Rev, B12(1975), 905. 